\title{
Intermixing and lateral composition modulation in GaAs/GaSb short period superlattices
}

\author{
C. Dorin and J. Mirecki Millunchick ${ }^{\mathrm{a})}$ \\ Department of Materials Science and Engineering, University of Michigan, Ann Arbor, \\ Michigan 48109-2136 \\ C. Wauchope \\ Electron Microbeam Analysis Laboratory, University of Michigan, Ann Arbor, Michigan 48109-2143
}

(Received 17 February 2003; accepted 21 May 2003)

\begin{abstract}
Lateral composition modulation on the group V sublattice has been investigated in GaAs/GaSb short period superlattices. The effect of As species and growth temperature on the appearance of lateral composition modulation was studied. Cross-sectional transmission electron microscopy and x-ray diffraction reciprocal space maps reveal that structures grown using As tetramers are always disordered, defective, and phase separated. Also, in these structures the As-rich regions appear to be composed of stacked GaAs quantum dots embedded in a GaSb matrix. The structures grown with As dimers show improved crystalline quality. Short period superlattices grown at $T<420{ }^{\circ} \mathrm{C}$ have flat interfaces and are laterally homogeneous, however, there is significant anion intermixing across the interfaces. Structures deposited at $420^{\circ} \mathrm{C}<T<445^{\circ} \mathrm{C}$ roughen during growth, and exhibit lateral composition modulation and anion intermixing. Growing at higher temperatures destroys both the superlattice structure and the lateral composition modulation. The As sticking coefficient was calculated and was found to range between $0.1 \leqslant \sigma \leqslant 0.17$ depending on the growth temperature and As species. (C) 2003 American Institute of Physics. [DOI: 10.1063/1.1591419]
\end{abstract}

\section{INTRODUCTION}

Mixed anion systems containing $\mathrm{Sb}$ are of great use in high-speed electronics and optoelectronic devices, such as infrared detectors, ${ }^{1}$ field effect transistors, ${ }^{2}$ and long wavelength lasers. ${ }^{3} \mathrm{GaAsSb}$, in particular, is a promising material for high-speed double heterojunction bipolar transistors. ${ }^{4}$ However, the material quality is a concern since the abruptness of the interfaces, the degree of intermixing, the anion incorporation, and the homogeneity of the material greatly affect the performance of these devices.

There has been a great deal of research investigating the interfacial control and the uniformity across the layers in mixed anion heterostructures. Zuo et al. used cross-sectional scanning tunneling microscopy to characterize the interfacial structure and compositional uniformity within $\mathrm{GaAs}_{x} \mathrm{Sb}_{1-x} / \mathrm{GaAs}$ double quantum well structures, ${ }^{5}$ and found that there was significant $\mathrm{Sb}$ segregation and clustering within the GaAsSb layer. These effects are strongly dependent on the growth conditions. Therefore, advanced growth techniques have been employed to overcome these problems, including migration enhanced epitaxy (MEE), growth interruptions, group V soaks, and V/III pressure ratio reductions. ${ }^{6-8}$

Another challenge with GaAsSb alloys is the difficulty in controlling the composition, which is strongly dependent on the growth temperature, group III growth rates and group $\mathrm{V}$ flux ratio. ${ }^{7}$ One common approach is the growth of digital

\footnotetext{
a)Electronic mail: joannamm@umich.edu
}

alloys. ${ }^{6}$ This method consists of depositing short period superlattices of, for example, $\mathrm{GaAs}_{n} / \mathrm{GaSb}_{m}$, where $n$ and $m$ are the number of monolayers (MLs) of the GaAs and GaSb, respectively. The volume fraction of the GaAs determines the As composition of the overall structure. This method has proven to accurately control the incorporation of the group $\mathrm{V}$ element, for compositions less than 50\%. ${ }^{6}$ Although the growth of digital alloys improved the accuracy in obtaining the desired composition, strain effects that may result in spontaneous phase separation, were not taken into account. This unintentional consequence may change the respective optical properties of the alloy such as band-gap and band offsets.

Lateral composition modulation is a type of phase separation that is perpendicular to the growth direction. It has been shown to be regular and robust for strain-balanced short period superlattices (SPS) where each layer is on the order of a monolayer or two, the ratio of the layer thicknesses is close to $1: 1$, and the layers have alternating compressive and tensile strain with respect to the substrate. ${ }^{9-14}$ A great deal of attention has been paid to mixed cation systems where the composition modulation occurs on the group III sublattice, such as GaAs/InAs, ${ }^{9}$ AlAs/InAs, ${ }^{10}$ and GaP/InP SPSs. ${ }^{11}$ Typical composition modulation wavelengths are on the order of $10 \mathrm{~nm}<\Lambda<40 \mathrm{~nm},{ }^{11}$ and the modulation amplitude varies in composition of $0.4<x<0.6 .^{12}$ Little has been reported to date regarding SPS structures where the composition modulation appears on the group $\mathrm{V}$ sublattice. ${ }^{13,14}$ In this article, we discuss the microstructure of GaAs/GaSb multilayers and describe the conditions for intermixing and lateral composition modulation in these films. 


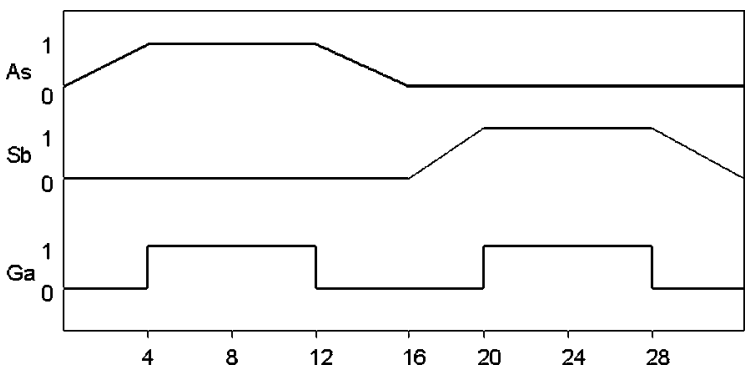

FIG. 1. Shuttering sequence used during the deposition of a typical SPS. 0 indicates a closed shutter and 1 indicates an open shutter.

\section{EXPERIMENTAL DETAILS}

All growths were carried out in a molecular beam epitaxy (MBE) chamber equipped with solid sources for Ga and In, and valved cracking cells for As and $\mathrm{Sb}$. The samples were grown upon $\operatorname{InP}(001)$ substrates that were prepared by heating to $530{ }^{\circ} \mathrm{C}$ under an $\mathrm{As}_{s}$ overpressure $(s=2$ or 4 , as noted). A nominally latticed matched InGaAs buffer layer was deposited at a temperature $T=500^{\circ} \mathrm{C}$. The SPSs were deposited at $370{ }^{\circ} \mathrm{C} \leqslant T \leqslant 460^{\circ} \mathrm{C}$ using $\mathrm{Sb}_{2}$ and $\mathrm{As}_{s}$. The $\mathrm{GaAs}$ and $\mathrm{GaSb}$ growth rates were 0.21 and $0.23 \mathrm{ML} / \mathrm{s}$, and were calibrated by reflection high energy electron diffraction (RHEED) oscillations on their respective substrates prior to growth of the structure. The ratio of $\mathrm{Sb}_{2}$ to $\mathrm{As}_{s}$ beam equivalent pressure (BEP) was approximately 0.3 and the total group V BEP was $10^{-5}$ Torr for all the samples in this study, except where noted. Switching between the group $\mathrm{V}$ species was achieved by both actuating the shutters and valves, taking into account the time necessary for the valves to open or close. A typical growth sequence is shown in Fig. 1. It should be noted that even though shuttering of the sources is very fast, actuating the valves of the group V sources to their full open or full closed positions takes on the order of $4 \mathrm{~s}$, as indicated in Fig. 1. Therefore, the valves were fully actuated prior to opening the Ga shutter. Several different multilayer structures were examined, as summarized in Tables I and III. In all instances the intended thickness was nominally $2 \mathrm{ML}$ for the GaSb layer and was either 1 or $2 \mathrm{ML}$ for the GaAs layer. Several $\mathrm{GaAs}_{x} \mathrm{Sb}_{1-x}$ alloy layers were grown under similar conditions as the SPS structures (Table II) for comparison.

The samples were examined by a variety of in situ and ex situ characterization techniques. The quality of the crystalline surface was monitored in situ by RHEED. X-ray diffraction (XRD) $\theta / 2 \theta$ scans and reciprocal space maps were recorded with $\mathrm{Cu} K \alpha$ radiation in order to characterize the
TABLE II. Summary of the $\mathrm{GaAs}_{x} \mathrm{Sb}_{1-x}$ alloy films, As species, As and $\mathrm{Sb}$ beam equivalent pressure and average composition $\langle x\rangle$ grown at $T$ $=400{ }^{\circ} \mathrm{C}$.

\begin{tabular}{ccccc}
\hline \hline Sample No. & & As BEP $\left(\times 10^{-6}\right.$ Torr $)$ & Sb BEP $\left(\times 10^{-6}\right.$ Torr $)$ & $\langle x\rangle$ \\
\hline G206 & $\mathrm{As}_{4}$ & 8 & 3 & 0 \\
$\mathrm{I} 404$ & $\mathrm{As}_{4}$ & 1.7 & 0.5 & 0 \\
$\mathrm{~K} 421$ & $\mathrm{As}_{2}$ & 8 & 3 & 0.52 \\
\hline \hline
\end{tabular}

microstructure of the SPS. Cross-sectional transmission electron microscopy (XTEM) was also performed. TEM samples were prepared using mechanical thinning followed by Ar-ion milling at $4.5 \mathrm{kV}$ and angles between $3^{\circ}$ and $5^{\circ}$. The buildup of In-metal islands on the surface was confined to the substrate and did not affect the sample quality in the region of interest. XTEM studies were carried out on a field emission TEM (JEOL 2010FX) operated at $200 \mathrm{kV}$. High angle annular dark field (HAADF) scanning transmission electron microscopy (STEM) imaging was used to collect images with high mass-thickness contrast and little to no diffraction contrast, allowing direct imaging of the composition modulation. $\mathrm{X}$-ray energy dispersive spectroscopy (XEDS) line profiles were collected using a software controlled STEM probe with drift correction.

\section{RESULTS}

\section{A. Growth under $\mathbf{A s}_{4}$}

In all cases, the growth of the GaAs/GaSb SPS structures using $\mathrm{As}_{4}$ resulted in rough and highly defective films. Nonetheless, lateral composition modulation was observed to some degree. The RHEED pattern for all the samples grown with $\mathrm{As}_{4}$ degraded into spots during growth, indicating threedimensional (3D) islanding. Typically, the RHEED pattern was spottier during the deposition of the GaAs layer but recovered to streaks that were modulated across their lengths during the deposition of the first GaSb layer. Although the surface reconstruction was $2 \times 4$ prior to the deposition of the SPS, it changed to $1 \times 3$ upon the deposition of the $\mathrm{GaSb}$ layer and remained so for the entire growth. Reducing the thickness of the GaAs layer to $1 \mathrm{ML}$, or performing a Sb soak prior to the deposition of the GaSb layer did not significantly improve the quality of the films.

(004) XRD $\theta / 2 \theta$ scans were performed in order to determine the percentage of $\mathrm{As}_{4}$ incorporated in the films. None of these structures show evidence of satellites arising from the SPS, indicating that the superlattice periodicity has been destroyed. This is not unexpected given that the RHEED

TABLE I. Summary of the $\mathrm{GaAs}_{n} / \mathrm{GaSb}_{m}$ structures grown under $\mathrm{As}_{4}$ overpressure, SPS average composition $\langle x\rangle$, full width at half maxima of the SPS Bragg peak (FWHM), average As composition in Sb rich regions $\left(x^{\mathrm{Sb}}\right)$, and As-rich regions $\left(x^{\mathrm{As}}\right)$ and lateral composition modulation wavelength $\Lambda$ for samples grown in this study at $T=400{ }^{\circ} \mathrm{C}$. * denotes an additional $4 \mathrm{~s} \mathrm{Sb}$ soak prior to the deposition of the GaSb layer.

\begin{tabular}{ccccccccc}
\hline \hline Sample No. & $n / m$ & $\langle x\rangle$ & FWHM $(")$ & $x^{\text {Sb }}$ & & $x^{\text {As }}$ & & $\Lambda(\mathrm{nm})$ \\
\hline F193 & $2 / 2$ & 0.45 & 192 & NA & NA & NA & NA & 20 \\
G226 & $1 / 2$ & 0.41 & 595 & 0.3 & \pm 0.07 & 0.6 & \pm 0.11 & 21 \\
G219 & $2 / 2^{*}$ & 0.42 & 359 & 0.3 & \pm 0.09 & 0.5 & \pm 0.06 & 24 \\
\hline \hline
\end{tabular}



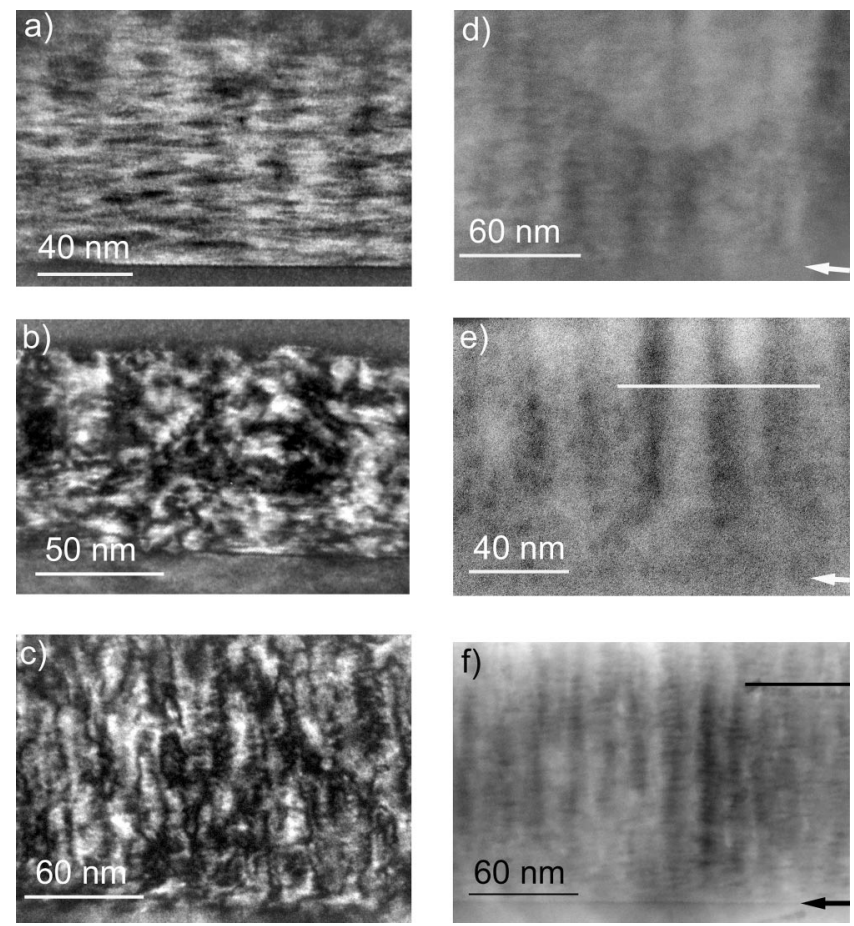

FIG. 2. (002) dark field XTEM images [(a)-(c)] and HAADF images [(d)(f)] for SPSs grown under $\mathrm{As}_{4}$ overpressure for the $\mathrm{GaAs}_{2} \mathrm{ML} / \mathrm{GaSb}_{2} \mathrm{ML}$ sample [(a) and (d)], $\mathrm{GaAs}_{1 \mathrm{ML}} / \mathrm{GaSb}_{2 \mathrm{ML}} \quad[(\mathrm{b})$ and (e)] and $\mathrm{GaAs}_{2 \mathrm{ML}} / \mathrm{GaSb}_{2 \mathrm{ML}} \mathrm{Sb}$ soak sample [(c) and (f)]. The arrows indicate the position of the interface.

pattern indicated surface roughening during growth. Also, consistent with this observation is that the width of the SPS Bragg peaks are fairly broad, with full width half maxima of $192 \leqslant$ FWHM $\leqslant 595$ arcseconds, indicating that the films contain a large density of defects. The measured As composition of the structures differs from the expected composition calculated from the nominal GaAs and GaSb layer thicknesses. For example, sample F193, which consists of 2 MLs each of $\mathrm{GaAs}$ and $\mathrm{GaSb}$, has an expected As composition of $x=0.5$ but was measured to be $x=0.45$. This discrepancy may be resolved by considering that the GaSb surface is terminated with between 1 7/12 and 1 2/3 MLs (Ref. 33) of excess Sb, which is incorporated into the structure as the GaAs layer is deposited, thus lowering the resulting As composition.

Incorporating a $4 \mathrm{~s} \mathrm{Sb}$ soak prior to the deposition of the $\mathrm{GaSb}$ layer resulted in further depletion of the As content, as demonstrated by the lower average As composition of this sample $(x=0.42)$. Decreasing the GaAs thickness to $1 \mathrm{ML}$ also lowered the average As composition to $x=0.41$ but not as much as expected $\left(x^{\text {expect }}=0.33\right)$.

The microstructure of the SPS was characterized by performing (002) XTEM dark field and HAADF imaging. (002) XTEM dark field micrographs of all samples [Figs. 2(a)-2(c)] show image contrast due to both strain and compositional variations across the layers. (002) dark field imaging has been proved to be useful in characterizing composition modulation in structures such as AlAs/InAs SPSs due to the fact that for the (002) diffraction condition, the structure factor and therefore the strain contrast, goes to zero for the $\operatorname{In}_{x_{0}} \mathrm{Al}_{1-x_{0}}$ As at a composition of $x_{0}=0.52$, which is

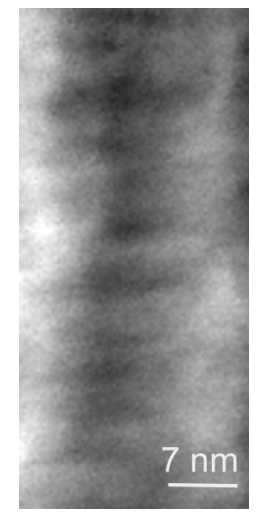

FIG. 3. HAADF image of an As-rich column in sample $\mathrm{GaAs}_{2 \mathrm{ML}} / \mathrm{GaSb}_{2 \mathrm{ML}} \mathrm{Sb}$ soak showing a column of vertically stacked 3D islands.

close to the experimental composition. Hence, the image contrast in that case is only due to compositional variations. ${ }^{12}$ However, this method cannot be used for the $\mathrm{GaAs} / \mathrm{GaSb}$ system because the structure factor goes to zero for $\mathrm{GaAs}_{x_{0}} \mathrm{Sb}_{1-x_{0}}$ at a composition of $x_{0}=0.1$, which is far from the compositions studied. Also, the strain in the GaAs/ GaSb samples has both shear and normal components due to presence of 3D islanding. Therefore, the strong contrast observed in the (002) dark field images is due primarily to strain with some contribution from compositional variations. In order to discern how the composition alone varies across the layers, HAADF images were acquired, since for this technique the image contrast is only due to differences in atomic mass and foil thickness. Lateral composition modulation is clearly visible for all the samples in HAADF images shown in Figs. 2(d)-2(f).

The microstructures of all three samples grown under $\mathrm{As}_{4}$ overpressure are very similar, with irregular lateral contrast variations and occasional threading dislocations. The dark regions in the HAADF images of Fig. 2 are enriched in As. At higher magnification these columns appear to be vertically stacked GaAs quantum dots embedded in a GaSb matrix, as shown for the $\mathrm{GaAs}_{2 \mathrm{ML}} / \mathrm{GaSb}_{2} \mathrm{ML}$ Sb soak sample in Fig. 3. This is consistent with the $3 \mathrm{D}$ islanding observed in the spotty RHEED pattern during the GaAs layer deposition.

X-ray energy dispersive spectroscopy was performed across the modulated regions of the samples in order to calculate the amplitude and wavelength of the composition modulation. XEDS line profiles at the positions marked in Figs. 2(e) and 2(f) are shown in Fig. 4. It is clear that the As and Sb compositions vary sinusoidally and out of phase with one another, confirming compositional variation on the group $\mathrm{V}$ sublattice. The wavelength of the modulated regions was obtained by fitting the line profiles with a sinusoidal function and was found to be approximately $21 \mathrm{~nm}$ for the $\mathrm{GaAs}_{1 \mathrm{ML}} / \mathrm{GaSb}_{2 \mathrm{ML}}$ sample, and approximately $24 \mathrm{~nm}$ for the $\mathrm{GaAs}_{2 \mathrm{ML}} / \mathrm{GaSb}_{2} \mathrm{ML} \mathrm{Sb}$ soak sample. The As compositions given in Table I for the Sb- and As-rich regions were averaged from multiple line profiles across different modulated regions, line profiles along the $\mathrm{Sb}$ - and As-rich regions, and XEDS compositional maps. The standard deviation is also given in Table I. For the $\mathrm{GaAs}_{1 \mathrm{ML}} / \mathrm{GaSb}_{2 \mathrm{ML}}$ sample 

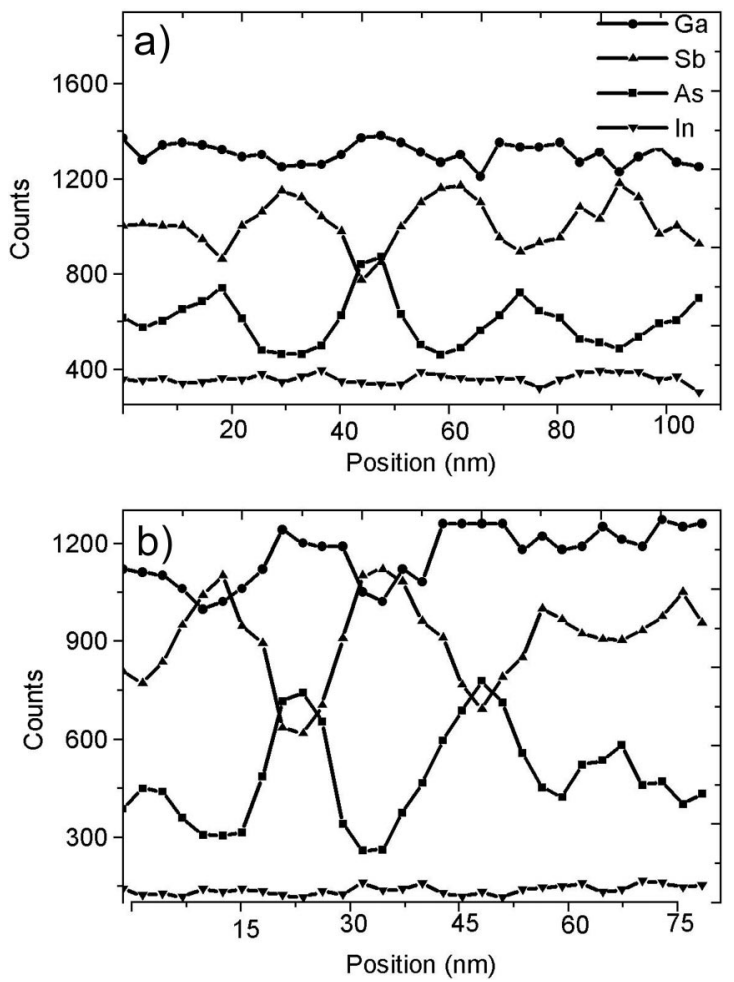

FIG. 4. XEDS line profiles for sample $\mathrm{GaAs}_{1 \mathrm{ML}} / \mathrm{GaSb}_{2 \mathrm{ML}}$ (a) and $\mathrm{GaAs}_{2} \mathrm{ML} / \mathrm{GaSb}_{2} \mathrm{ML}$ Sb soak (b) for the lines indicated in Figs. 3(e) and 3(f). As and $\mathrm{Sb}$ compositions are oscillating of phase with each other.

and the $\mathrm{GaAs}_{2 \mathrm{ML}} / \mathrm{GaSb}_{2 \mathrm{ML}} \mathrm{Sb}$ soak sample the average As composition in the Sb-rich regions is the same, $x^{\mathrm{Sb}}=0.3$. However, the two samples have different As compositions in the As rich regions: $x^{\mathrm{As}}=0.5$ for the $\mathrm{GaAs}_{2 \mathrm{ML}} / \mathrm{GaSb}_{2 \mathrm{ML}} \mathrm{Sb}$ soak sample compared to $x^{\mathrm{As}}=0.6$ in the $\mathrm{GaAs}_{1 \mathrm{ML}} / \mathrm{GaSb}_{2 \mathrm{ML}}$ sample, suggesting that the soak step inhibits As incorporation. Figure 5(a) shows the HAADF image of the $\mathrm{GaAs}_{1 \mathrm{ML}} / \mathrm{GaSb}_{2 \mathrm{ML}}$ sample in the region where the Sb map shown in Fig. 5(b) was taken. Sb x-ray counts collected at each pixel position are represented on a gray scale where white represents high counts. The x-ray map for As is the inverse of the Sb map, showing again lateral composition modulation of the group $\mathrm{V}$ elements. Although the XEDS map was collected with a drift corrected STEM probe, some drifting is apparent in the wavy columns of $\mathrm{Sb}$ in the map, compared to the straight columns in the HAADF image.
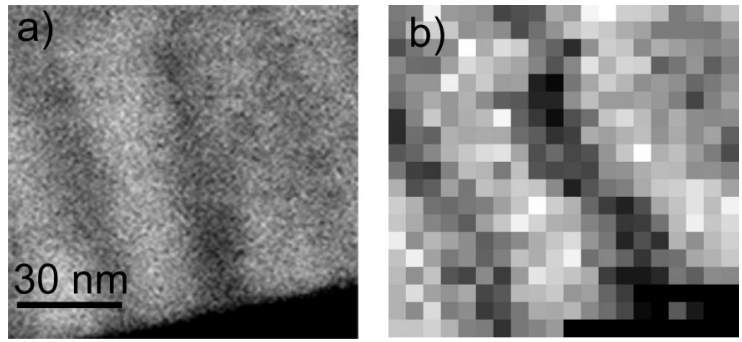

FIG. 5. HAADF image of a modulated region for $\mathrm{GaAs}_{1 \mathrm{ML}} / \mathrm{GaSb}_{2} \mathrm{ML}$ sample (a) and the corresponding XEDS compositional map (b). The white regions are enriched in $\mathrm{Sb}$.
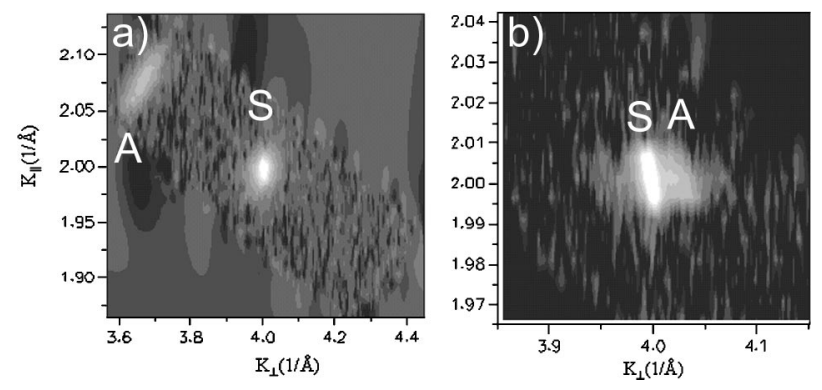

FIG. 6. (224) reciprocal space maps for the $\mathrm{GaAs}_{x} \mathrm{Sb}_{1-x}$ alloys deposited with $\mathrm{As}_{4}$ (a) and $\mathrm{As}_{2}$ (b). $S$ and $A$ indicate the substrate and the alloy Bragg peaks.

Also apparent in Fig. 4 is a small oscillation in the Ga composition in phase with the As oscillation. The amplitude of the oscillation is on the order of $1-4 \mathrm{wt} \%$, depending on the sample. One possible explanation is that In from the InGaAs buffer layer segregated into the layer and subsequently phase separates from the Ga on the group III sublattice. However, no In was found in the SPSs according to XEDS. Furthermore, In segregation is negligible at this growth temperature $\left(T=400{ }^{\circ} \mathrm{C}\right)$. Another possible explanation is that the Ga compositional oscillations are due to excess $\mathrm{Sb}$ atoms sitting in interstitial sites or occupying $\mathrm{Ga}$ sites. $^{15}$

Random GsAs $\mathrm{Sb}_{1-x}$ alloys (Table II) were grown in order to determine whether the lateral composition modulation is simply a result of spinodal decomposition. The XRD (224) reciprocal space map for a $\mathrm{GaAs}_{x} \mathrm{Sb}_{1-x}$ film deposited at the same conditions as the $\mathrm{GaAs}_{2 \mathrm{ML}} / \mathrm{GaSb}_{2 \mathrm{ML}}$ SPS [Fig. 6(a)], shows that the film peak is broadened along $\omega$ indicating that the film has a large density of dislocations, ${ }^{16,17}$ however, there are no lateral satellites, suggesting that the alloy is structurally homogeneous. (224) glancing incidence and glancing exit XRD measurements show that the alloy is fully relaxed and that $x=0$, i.e., no As incorporation has occurred. XTEM also showed a homogeneous film with defects and the absence of As was confirmed by XEDS (not shown). $\mathrm{XRD}$ rocking curves indicate that the tilt in this sample is 43 arcseconds away from the (001) plane, too small to be a primary mechanism responsible for strain relaxation in this case. Another $\mathrm{GaAs}_{x} \mathrm{Sb}_{1-x}$ random alloy was grown at the same temperature and $\mathrm{Sb} / \mathrm{As}$ BEP ratio, but at a lower total group $\mathrm{V}$ overpressure $\left(2 \times 10^{-6}\right.$ Torr $)$ in order to take into account the lower instantaneous group $\mathrm{V}$ fluxes that are present during the deposition of the multilayer structure. No As incorporation occurred in this film either. This observation is consistent with reports that have shown that the As incorporation is greatly reduced in the presence of $\mathrm{Sb}$ at high group V overpressure. ${ }^{18}$ Furthermore, the cracking of $\mathrm{As}_{4}$ in the presence of $\mathrm{Sb}_{2}$ flux is inefficient at these low growth temperatures $\left(T=400{ }^{\circ} \mathrm{C}\right) .{ }^{7}$

\section{B. Growth under $A s_{2}$}

In order to increase the As incorporation and improve the quality of these films, $\mathrm{As}_{2}$ was used in place of the $\mathrm{As}_{4}$ flux. ${ }^{7}$ Figure 6(b) shows the (224) reciprocal space map of a 
TABLE III. Summary of the $\mathrm{GaAs}_{n} / \mathrm{GaSb}_{m}$ SPS samples grown under an $\mathrm{As}_{2}$ overpressure for various growth temperature $T$, observed GaAs $\left(n^{\circ}, y\right)$ and GaSb $\left(m^{\circ}, z\right)$ thickness and As composition, average SPS composition $\langle x\rangle$, full with at half maxima of the SPS Bragg peak (FWHM), As composition in the $\mathrm{Sb}$ rich regions $\left(x^{\mathrm{Sb}}\right)$ and As-rich regions $\left(x^{\mathrm{As}}\right)$ modulated regions and modulation wavelength $\Lambda$.

\begin{tabular}{|c|c|c|c|c|c|c|c|c|c|c|c|c|}
\hline Sample No. & $T\left({ }^{\circ} \mathrm{C}\right)$ & $n^{\mathrm{o}}$ & $m^{\circ}$ & $y$ & $z$ & $\langle x\rangle$ & FWHM & $x^{\mathrm{Sb}}$ & & $x^{\text {As }}$ & & $\Lambda(\mathrm{nm})$ \\
\hline L609 & 370 & 2.15 & 2.33 & 0.62 & 0.32 & 0.48 & 143 & & & No CM & & \\
\hline K424 & 400 & 2.12 & 2.26 & 0.6 & 0.4 & 0.48 & 155 & & & No CM & & \\
\hline K449 & 420 & 2.12 & 2.2 & 0.58 & 0.38 & 0.49 & 177 & & & No CM & & \\
\hline K446 & 430 & 2.4 & 1.9 & 0.65 & 0.35 & 0.55 & & 0.5 & \pm 0.02 & 0.7 & \pm 0.07 & 15 \\
\hline K448 & 445 & 2.3 & 2 & 0.7 & 0.33 & 0.53 & & 0.5 & \pm 0.03 & 0.6 & \pm 0.04 & 23 \\
\hline K447 & 460 & NA & NA & NA & NA & NA & & 0.5 & \pm 0.06 & 0.7 & \pm 0.05 & 20 \\
\hline
\end{tabular}

GaAsSb alloy grown with $\mathrm{As}_{2}$ under the same conditions as the $\mathrm{As}_{4}$ film shown in Fig. 6(a). Peaks due to substrate and alloy are present in the map, and the As composition of the layer was found to be $x=0.52$. The lack of lateral satellites along the [110] direction show that this alloy is uniform, confirming that lateral composition modulation observed in the SPS structures is not due to spinodal decomposition, despite the presence of a miscibility gap in GaAsSb alloys at this growth temperature and for these compositions. ${ }^{16}$

The structures grown under an $\mathrm{As}_{2}$ overpressure, summarized in Table III, show marked improvements in crystal quality. Samples grown at low temperature, $370 \leqslant T$ $\leqslant 420^{\circ} \mathrm{C}$, exhibited layer-by-layer growth as observed by monitoring the RHEED specular spot intensity as a function of time. Furthermore, the RHEED pattern remained streaky over the entire growth and the surface reconstruction remained $1 \times 3$. Figure $7(a)$ is the (224) reciprocal space map for a sample grown at $T=420{ }^{\circ} \mathrm{C}$. Peaks due to the substrate, buffer layer, and SPS peak are clearly visible, and no lateral satellites are detected. The average As composition for this sample is $x=0.48$. Figure 7(b) is a (002) dark field XTEM micrograph that shows the SPS individual layers. Moire fringes are occasionally observed in these images, and are due to beam damage during TEM imaging. ${ }^{19}$ HAADF imaging confirmed that there was no lateral composition modulation.

For samples grown at $430^{\circ} \mathrm{C} \leqslant T \leqslant 445^{\circ} \mathrm{C}$, the RHEED pattern changed during deposition from streaky to spotty at a thickness dependent on the growth temperature. For the sample grown at $T=430{ }^{\circ} \mathrm{C}$, the streaks in the RHEED pattern became modulated across their length towards the end of

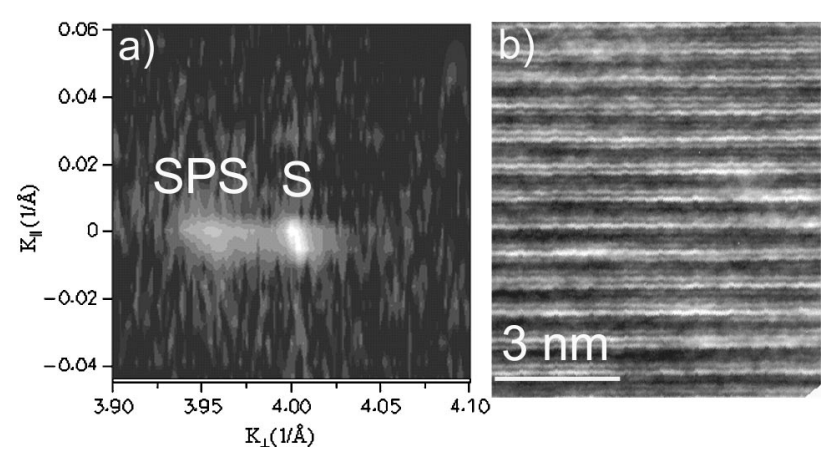

FIG. 7. (224) reciprocal space map (a) and (002) dark field XTEM (b) for $\mathrm{GaAs}_{2 \mathrm{ML}} / \mathrm{GaSb}_{2 \mathrm{ML}}$ sample grown under $\mathrm{As}_{2}$ overpressure at $T=420^{\circ} \mathrm{C} . S$ is the Bragg peak due to the substrate and buffer layer and SPS is the Bragg peak due to the SPS. the deposition. Figure 8(a) is the (224) reciprocal space map of this sample, and shows peaks due to substrate, buffer layer and the SPS. Also visible in this image are weak satellites along the [110] direction indicating that the sample possesses some lateral composition modulation. The average As composition of the overall structure is $x=0.55$ in this case. (002) dark field XTEM imaging [Fig. 8(b)] confirms the presence of irregular lateral composition modulation, however, it is interesting to note that the modulation profile is asymmetric. That is, the Sb-rich regions are approximately five times wider than the As rich regions.

The RHEED pattern for the sample grown at $T$ $=445^{\circ} \mathrm{C}$ became spotty after approximately 80 periods were deposited. Lateral composition modulation $(\mathrm{CM})$ was observed in HAADF images (not shown) with the same asymmetry in the modulation profile. The asymmetry of the modulated regions is more pronounced in the plan-view HAADF image of this sample [Fig. 9(a)]. The Sb-rich regions (light areas) form a cellular structure delineated by narrow (dark) As-rich regions. An XEDS line profile [Fig. 9(b)] across a modulated region again shows that As and $\mathrm{Sb}$ oscillate out of phase. The wavelength is approximately 15 $\mathrm{nm}$, and the As composition is $x^{\mathrm{Sb}}=0.5$ in the Sb-rich regions and $x^{\mathrm{As}}=0.6$ in the As-rich regions.

Structures grown at higher temperatures, $T=460^{\circ} \mathrm{C}$, began to degrade according to RHEED after approximately ten periods of deposition. At the end of growth, the RHEED pattern was very dim and showed chevrons indicating the presence of 3D faceting. Figure 10(a) is the (002) XTEM bright field image of this sample. Lateral CM and the SPS individual layers are visible near the buffer-SPS interface. However, those features are obscured at approximately 40 $\mathrm{nm}$ (or nominally 30 periods) and the film appears to become

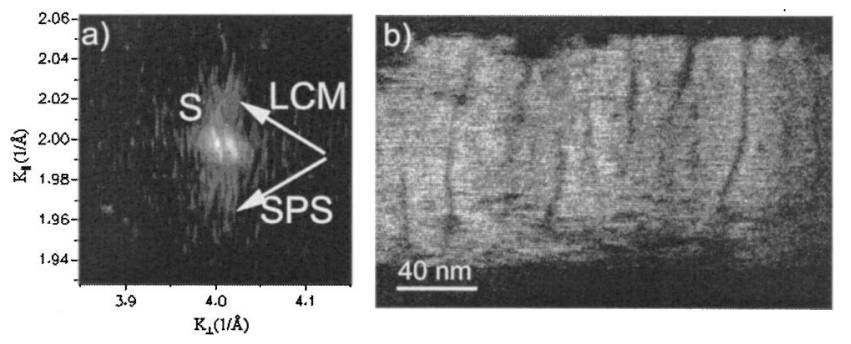

FIG. 8. (224) reciprocal space map (a) and (002) dark field XTEM (b) for the $\mathrm{GaAs}_{2} \mathrm{ML} / \mathrm{GaSb}_{2} \mathrm{ML}$ sample grown under $\mathrm{As}_{2}$ overpressure at $T$ $=430{ }^{\circ} \mathrm{C}$. The satellites along the [110] directions in (a) are due to lateral composition modulation (LCM). The dark regions in (b) are enriched in As. 

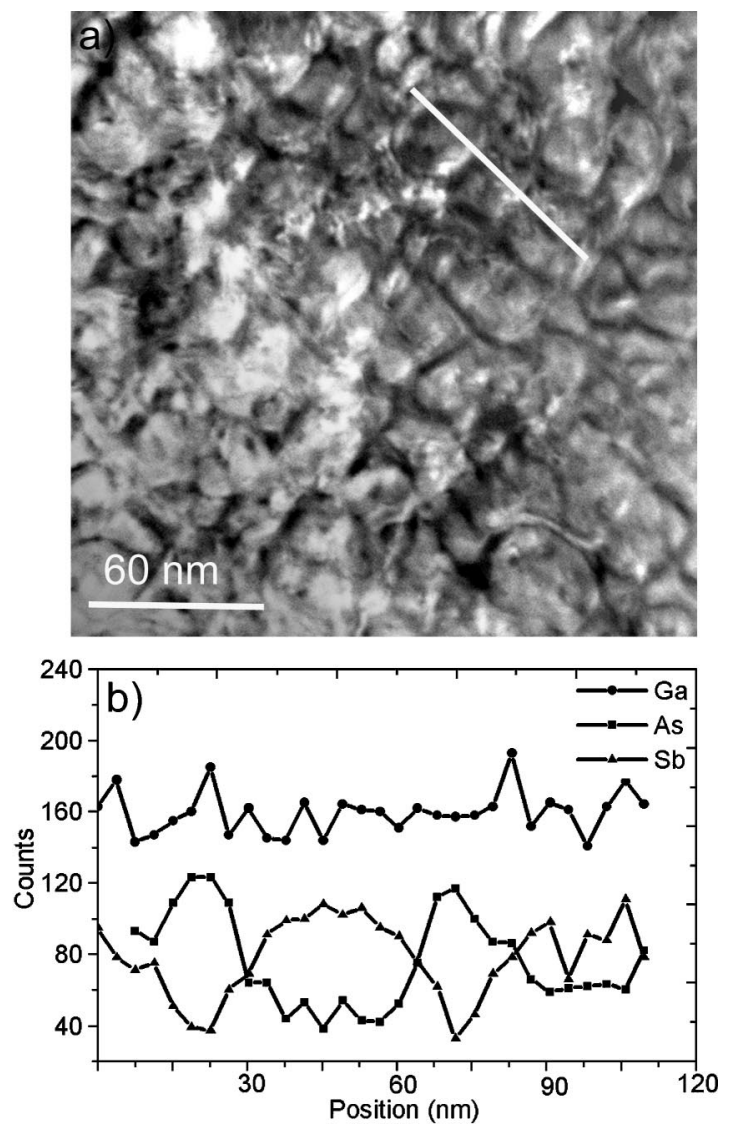

FIG. 9. Plan view HAADF image (a) and XEDS line profile for the line in a (b) for $\mathrm{GaAs}_{2} \mathrm{ML} / \mathrm{GaSb}_{2}$ ML sample grown under $\mathrm{As}_{2}$ overpressure at $T$ $=445{ }^{\circ} \mathrm{C}$.

intermixed and defective, with large facets on the surface. The HAADF image of this sample [Fig. 10(b)] shows regular and periodic composition modulation near the buffer-SPS interface, with As compositions of $x^{\mathrm{Sb}}=0.5$ in Sb-rich regions and $x^{\mathrm{As}}=0.7$ in As-rich regions [Fig. 10(c)]. In the upper part of the film, large-scale contrast variations in the HAADF image reveal that the remainder of the film is not homogeneous in composition but, according to XEDS analysis, varies in As composition between $0.25 \leqslant x \leqslant 0.75$. Surprisingly, Ga also varies in composition from $0.46 \leqslant x_{\mathrm{Ga}}$ $\leqslant 0.52$ within this region, as shown in Fig. 10(d).

\section{DISCUSSION}

These experiments clearly show that As species and growth temperature are dominant factors in producing high quality structures. This is not surprising because it has been shown that tetramer, dimer or monomer As species incorporate at different rates in III-V compound semiconductors. ${ }^{20}$ It has also been reported that $\mathrm{As}_{s}$ incident on the surface in the presence on an $\mathrm{Sb}$ flux leads to an anion exchange reaction, in which As displaces $\mathrm{Sb}$ atoms from the surface, that is strongly dependent on the growth temperature and flux ratios. ${ }^{21,22}$ The fact that the $\mathrm{As}_{2}$ samples are higher crystalline quality is not unexpected, since it has been well established that $\mathrm{As}_{2}$ is more reactive on GaSb surfaces compared to $\mathrm{As}_{4}$, resulting in higher As incorporation in structures
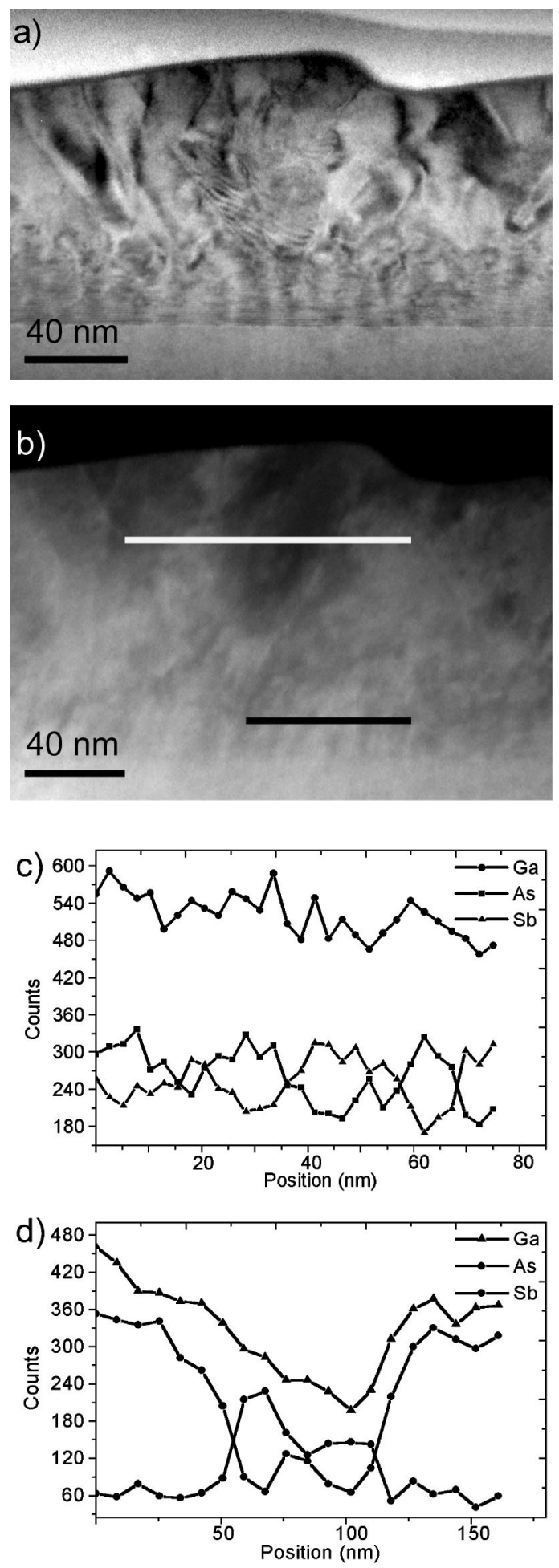

FIG. 10. (002) BF XTEM image (a), HAADF image (b) showing lateral composition modulation for approximately 30 periods of the SPS, and XEDS line profiles across the black line in $\mathrm{b}$ (c) and white line in $\mathrm{b}$ (d) for $\mathrm{GaAs}_{2 \mathrm{ML}} / \mathrm{GaSb}_{2 \mathrm{ML}}$ sample grown under $\mathrm{As}_{2}$ overpressure at $T=460{ }^{\circ} \mathrm{C}$.

containing $\mathrm{As}_{2} \cdot{ }^{23}$ This leads to better lattice matching of the structure to the InP substrate, thus, smoother films.

Structures grown using $\mathrm{As}_{4}$ exhibit significant roughening on the GaAs layer. A similar result was also observed in InAs/GaSb, where multilayer structures grown with $\mathrm{As}_{4}$ resulted in undulation of the InAs layer. ${ }^{24,25}$ It is possible that 


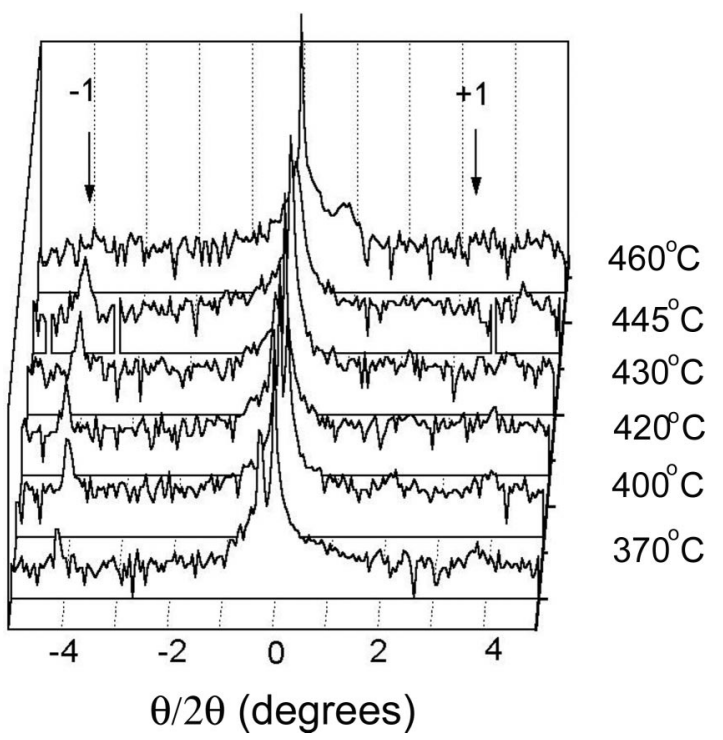

FIG. 11. (004) $\theta / 2 \theta$ XRD scans for the samples in Table III. The arrows indicate the position of the first order satellites due to the periodicity of the SPS.

this roughening is a result of reduced As incorporation and the resulting lattice mismatch. However, the deviation away from lattice matching of the $\mathrm{As}_{4}$ structures $(\approx-0.5 \%)$ is not large enough to account for all of the observed roughening. Therefore, it is likely that the interfacial instabilities observed in these structures are also due to differences in surface energies, surface reconstruction and/or surface diffusivities between the surfaces exposed to $\mathrm{As}_{2}$ compared to those exposed to $\mathrm{As}_{4}$.

In order to study the As incorporation as a function of temperature, (004) $\theta / 2 \theta$ scans were performed for the SPS structures grown under an $\mathrm{As}_{2}$ overpressure. Figure 11 plots the (004) $\theta / 2 \theta$ scans for the samples tabulated in Table III. The first-order SPS satellites are clearly visible for all the samples except the highest temperature one, which showed significant disordering of the SPS structure. The intensity and position of the first order satellites peaks were fitted with RADS Mercury commercial software in order to calculate the actual SPS individual layer thickness $(n, m)$ and the composition ( $y$ and $z$ ). These values are given in Table III. In most cases, the SPS individual layers are thicker than intended ( $2 \mathrm{ML}$ ), which may be due to the shuttering sequence that was used during growth. That is, additional material may have incorporated during the period of time that the valves were actuated. On the other hand, the thicker SPS individual layers thicknesses may be due to incorporation of excess $\mathrm{Sb}$ associated with the surface reconstruction of $\mathrm{GaSb}^{33}$ From the XRD data, it is also clear that the average As composition, thus the As incorporation, increases with increasing temperature, consistent with other reports. ${ }^{7}$ This might be attributed to the lower $\mathrm{Sb}$ vapor pressure compared to As, causing a larger fraction of the $\mathrm{Sb}$ to desorb from the surface at higher temperatures. It is also interesting to note that the quality of the films, as indicated by the FWHM, deteriorates with decreasing As content, consistent with the fact that these films are more strained.

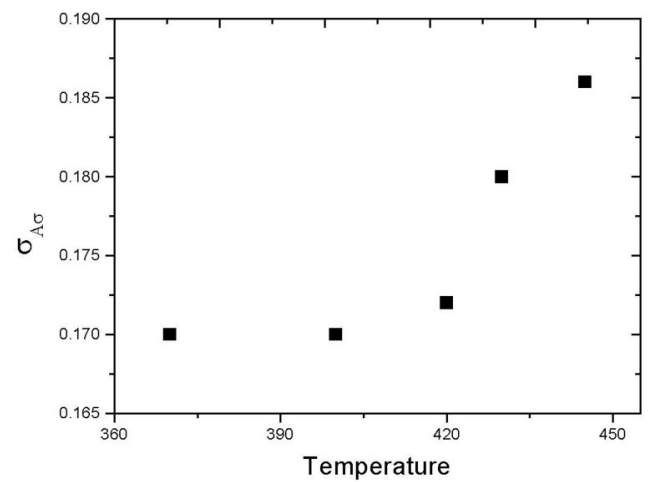

FIG. 12. $\mathrm{As}_{2}$ sticking coefficient vs temperature.

These data show that the As sticking coefficient is strongly temperature dependent. The As sticking coefficient for a GaAs/GaSb SPS can be quantitatively estimated by using the relation

$$
\langle x\rangle=\frac{F_{\mathrm{As}}}{F_{\mathrm{Ga}}} \times \sigma_{\mathrm{As}} \times \frac{n}{n+m},
$$

where $F_{\mathrm{As}}$ is the $\mathrm{As}_{2}$ growth rate expressed in $\mathrm{ML} / \mathrm{s}, F_{\mathrm{Ga}}$ is the $\mathrm{Ga}$ growth rate expressed in ML/s, $x$ is the average As composition as determined from (004) XRD $\theta / 2 \theta$ scans, $\sigma_{\mathrm{As}}$ is the As sticking coefficient, $n$ is the thickness of the GaAs layer, and $m$ is the thickness of the GaSb layer. This equation may be used despite the significant As-Sb intermixing because the $\mathrm{As}$ and the $\mathrm{Sb}$ fluxes are not competing against each other. In other words, the As and Sb shutters are opened and closed alternatively. $F_{\text {As }}$ was experimentally determined to be $1.25 \mathrm{ML} / \mathrm{s}$ by performing group V RHEED oscillations at the appropriate As overpressure. Figure 12 shows a plot of $\sigma_{\mathrm{As}_{2}}$ as a function of temperature. $\sigma_{\mathrm{As}_{2}}$ is a constant $\left(\sigma_{\mathrm{As}_{2}}\right.$ $=0.17$ ) for growth temperatures $370 \leqslant \mathrm{~T} \leqslant 420^{\circ} \mathrm{C}$. Over this temperature range, the SPS is in compression with respect to the InP substrate, the growth regime is layer by layer and no lateral composition modulation is observed. At temperatures $420^{\circ} \mathrm{C} \leqslant T \leqslant 460^{\circ} \mathrm{C}, \sigma_{\mathrm{As}_{2}}$ increases with temperature. This also corresponds to films that are more tensile, rougher, and compositionally modulated. It is not clear whether the increase in $\sigma_{\mathrm{As}_{2}}$ is due to the increase in roughening or another mechanism, such as decreasing Sb surface coverage, for example. It is possible that $\mathrm{As}_{2}$ is more reactive in the presence of $\mathrm{Sb}_{2}$ on a surface with a high density of steps and kinks.

The sticking coefficient for the $\mathrm{As}_{4}$ growths, which were all conducted at $T=400{ }^{\circ} \mathrm{C}$ and $F_{\mathrm{As}_{4}}=2 \mathrm{ML} / \mathrm{s}$, was calculated using the same approach. The sticking coefficient for both $\mathrm{GaAs}_{2 \mathrm{ML}} / \mathrm{GaSb}_{2 \mathrm{ML}}$ samples is $\sigma_{\mathrm{As}_{4}}=0.11$, which is smaller compared to $\sigma_{\mathrm{As}_{2}}=0.17$ for the analogous $\mathrm{As}_{2}$ sample. It is possible that the deviation from the intended thickness of the individual layers, i.e., $n$ and $m$ are not $2 \mathrm{ML}$ each, to be responsible for this difference in the sticking coefficient. The sticking coefficient for the $\mathrm{GaAs}_{1 \mathrm{ML}} / \mathrm{GaSb}_{2 \mathrm{ML}}$ sample was found to be $\sigma_{\mathrm{As}_{4}}=0.15$, larger than $\sigma_{\mathrm{As}_{4}}$ for $\mathrm{GaAs}_{2 \mathrm{ML}} / \mathrm{GaSb}_{2 \mathrm{ML}}$. The fact that $\sigma_{\mathrm{As} 4}$ depends on the amount of GaAs deposited suggests that it is strongly dependent on the fraction of $\mathrm{Sb}$ at the surface. 
The most regular lateral composition modulation was observed in films that were grown in tension, consistent with previous reports. ${ }^{12}$ For example, lateral composition modulation was observed in only those $\mathrm{As}_{2}$ samples that were in tension with respect to the substrate, and not in samples that were in compression. All the $\mathrm{As}_{4}$ structures were grown in compression with respect to the substrate, but exhibited significantly irregular lateral composition. It was not possible to grow the $\mathrm{As}_{4}$ samples in tension because of reduced As incorporation at these growth conditions.

The intermixing due to $\mathrm{Sb}$ surface segregation also alters the interlayer strain, which has significant impact on the final microstructure. ${ }^{26,27}$ If there is no segregation, for example, the mismatch $f$ between the individual GaAs and GaSb layers is $7 \%$. In reality there is some intermixing due to $\mathrm{Sb}$ surface segregation, resulting in an SPS consisting of $\mathrm{GaAs}_{y} \mathrm{Sb}_{1-y} / \mathrm{GaAs}_{z} \mathrm{Sb}_{1-z}$ layers, and the interlayer mismatch is proportionately lower. Figure 12 shows a plot of the observed interlayer mismatch as a function of growth temperature. Those samples that exhibit lateral composition modulation are denoted by open symbols. The interlayer mismatch is a minimum near $T=400{ }^{\circ} \mathrm{C}$, and lateral composition modulation only appears in samples grown at $T$ $=430^{\circ} \mathrm{C}$.

The average As composition in the individual superlattice layers, thus the mismatch between the layers, may be modeled using an approach first introduced by Dehaese et $a l^{28}$ Segregation is determined by atomic exchange between the surface layer and the first subsurface layer, assuming only layer-by-layer growth. The evolution of the number of $\mathrm{Sb}$ surface atoms is given by the balance of incoming and leaving $\mathrm{Sb}$ atoms. The exchange process of $\mathrm{Sb}$ atoms from the bulk to the surface is achieved by overcoming an energy barrier $E_{1}$ with a rate $\nu \exp \left(-E_{1} / k T\right)$, where $\nu$ is a vibration frequency on the order of $10^{13} \mathrm{~s}^{-1}, k$ is the Boltzman constant, and $T$ is the growth temperature. The reverse exchange (from the surface to the bulk) is also possible. That energy barrier is $E_{1}+E_{s}$, where $E_{s}$ is the segregation energy, and has a rate $\nu \exp \left[-\left(E_{1}+E_{s}\right) / k T\right]$. Assuming that segregation is due only to this exchange process, the number of the $\mathrm{Sb}$ surface atoms is given by the equality of the incoming and leaving $\mathrm{Sb}$ atoms, conserving the number of $\mathrm{Sb}$ atoms and total surface atoms as a function of time. The Sb concentration profile can be built by numerically solving these expressions, from which the interlayer mismatch may be calculated. Figure 13 plots the observed and the theoretical interlayer mismatch versus temperature for $E_{1}=1.85 \mathrm{eV}$ and $E_{s}=0.08 \mathrm{eV} .{ }^{29}$ There is a minimum in the interlayer strain at $T=400{ }^{\circ} \mathrm{C}$, and away from that temperature the interlayer strain increases.

The position of the minimum could be replicated using the model, however, the exact shape of the curve could not. Perhaps the most severe limitation of the model is that it assumes that $\mathrm{Sb}$ exchange occurs only between the surface and the first sublayer. In these films, there is also significant roughening, which may open other paths for $\mathrm{Sb}$ segregation. Also, the model does not consider the effect of the surface reconstruction or interaction with the vapor phase. Nonetheless, the model qualitatively agrees with the experimental

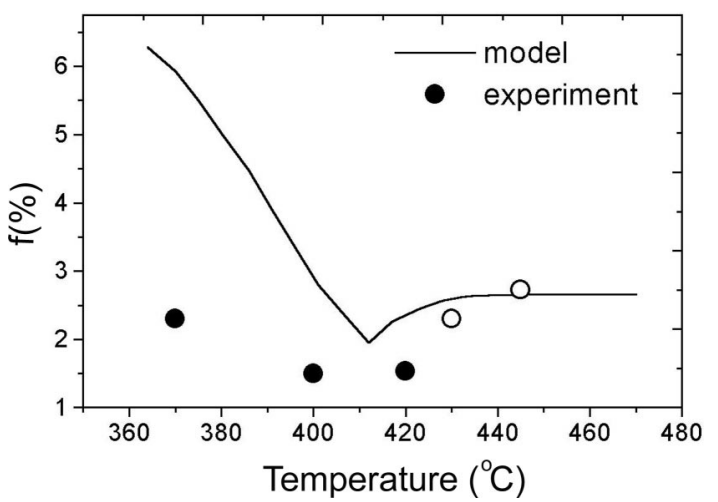

FIG. 13. SPS individual layers mismatch $\mathrm{f}$ versus temperature from experiment and using the kinetic model. The solid line is the mismatch using the kinetic model for barrier energy $E_{1}=1.85 \mathrm{eV}$ and segregation energy $E_{s}$ $=0.08$. The circles are the experimental mismatch. The solid circles indicate samples without lateral composition modulation and the open circles indicate that the samples possess lateral composition modulation.

data, namely that the interlayer strain is minimized at $400^{\circ} \mathrm{C}$

Lateral composition modulation appears in structures where the thickness of the individual SPS layers is on the order of the critical thickness for roughening, which depends both on the interlayer strain and on the growth temperature. Lateral composition modulation does not occur when the growth is layer by layer. $^{30}$ Indeed, films grown at $T$ $<420^{\circ} \mathrm{C}$ did grow layer by layer, as indicated by RHEED oscillations and no lateral composition modulation was observed, despite the fact that the interlayer strain is relatively high. However, when the films roughened during growth, lateral composition modulation was observed. In this case, the kinetic critical thickness for roughening is on the order of the individual SPS layer. Too much roughening destroys the regularity of the lateral composition modulation, as observed in the sample grown at $T=460{ }^{\circ} \mathrm{C}$. In that case, the integrity of the SPS itself broke down after ten periods of deposition.

It is interesting to note that for $\mathrm{As}_{4}$ samples, the As-rich regions appear to be composed of stacked GaAs quantum dots embedded in a GaSb matrix (see Fig. 3). In the planview HAADF image of $\mathrm{As}_{2}$ sample K448 (Fig. 9) the cells/ columns are also GaAs in a surrounding matrix of GaSb. This phenomenon is perhaps unexpected, since in most of the reports of vertically stacked quantum dots, the material that comprises the quantum dot is in compression (InAs/ $\mathrm{GaAs}$ is the most common example). This phenomenon may be explained using perturbation theory as applied to strain balanced multilayer structures. ${ }^{31}$ According to this model, interface undulations in multilayer films are related to elastic instability of the free surface. For example, it is well established that a compressive film (e.g., GaSb on InP) is unstable with respect to perturbations in the surface planarity. The perturbation may either decay or continue to grow when a subsequent tensile layer is deposited. In the case of continued instability, the undulations on the compressive and tensile films may be either in phase or out of phase [see Fig. 3 in Ref. 31], depending on the relative layer thickness and 
diffusion length. The out-of-phase condition results in lateral composition modulation. In this work, a spotty RHEED pattern shows that an initial perturbation is initiated on the GaAs layer, which is also the first deposited layer. RHEED observations indicated smoothening of the film with the deposition of the GaSb layer. This could be due to a perturbation in the $\mathrm{GaSb}$ of equal amplitude and $180^{\circ}$ out of phase, thus resulting in a flat layer. It is also possible that the smoothening of the GaSb layer is due to kinetic effects associated with the relative growth rate of $\mathrm{GaSb}$ along the undulated GaAs surface. It has been shown that GaSb has a higher growth rate on high index surfaces ${ }^{32}$ leading to a tendency for any undulations to become filled. Vertically stacked quantum dots in the tensile phase have also been observed in InAs/GaSb superlattices. ${ }^{25}$

\section{CONCLUSIONS}

The microstructure of $\mathrm{GaAs}_{n} / \mathrm{GaSb}_{m}$ short period superlattices deposited on (001) InP substrates grown by molecular beam epitaxy was investigated as a function of As species and growth temperature. Structures grown with $\mathrm{As}_{4}$ and 1 $<n<2$ ML and $m=2 \mathrm{ML}$ exhibit significant roughening during growth. These films are highly defective but HAADF images reveal the presence of composition modulation perpendicular to the growth direction. Surprisingly, the Ga composition varies laterally as well, suggesting that antisite defects may be present. Also in these structures, the As-rich regions appear to be composed of stacked GaAs quantum dots embedded in a GaSb matrix. Replacing As tetramers with As dimers improves the crystalline quality. Samples grown at $T \leqslant 420^{\circ} \mathrm{C}$ exhibit layer-by-layer growth as monitored by RHEED. The resulting superlattices are regular and laterally homogeneous. As growth temperature is increased, some roughening occurs and lateral composition modulation is formed. At growth temperatures $T>445{ }^{\circ} \mathrm{C}$ the SPSs became very rough, destroying both the vertical and lateral periodicity. For the samples grown under an $\mathrm{As}_{2}$ overpressure, the lateral composition modulation profile is asymmetric. The As sticking coefficient was found to be strongly dependent on temperature and As species, with some indication that the $\mathrm{Sb}$ coverage also plays a role. The variation in the microstructure with increasing temperature may be explained by taking $\mathrm{Sb}$ surface segregation into account. The intermixing between the individual SPS layers induced by $\mathrm{Sb}$ segregation alters the lattice mismatch between the layers, thus the growth mode. When the growth mode is layer by layer, lateral composition modulation is not observed. Lateral composition modulation is present for films that roughen during growth (see, also, Ref. 33).

\section{ACKNOWLEDGMENTS}

The authors acknowledge helpful discussions with $\mathrm{M}$. Bouville and Dr. B. Bennett. This work is supported by NSF Grant No. DMR 9973352.

${ }^{1}$ F. Fuchs, U. Weimer, W. Pletschen, J. Schmitz, E. Ahlswede, M. Walther, and J. Wagner, Appl. Phys. Lett. 71, 3251 (1997).

${ }^{2}$ C. R. Bolognesi, E. J. Caine, and H. Kroemer, IEEE Electron Device Lett. 15, 16 (1994).

${ }^{3}$ J. F. Klem, O. Blum, S. R. Kurtz, I. J. Fritz, and K. D. Choquette, J. Vac. Sci. Technol. B 18, 1605 (2000).

${ }^{4}$ C. R. Bolognesi, Compound Semicond. 6, 94 (2000).

${ }^{5}$ S. L. Zuo, Y. G. Hong, E. T. Yu, and J. F. Klem, J. Appl. Phys. 92, 3761 (2002).

${ }^{6}$ Y.-H. Zhang, J. Cryst. Growth 150, 838 (1995).

${ }^{7}$ B. R. Bennett, B. V. Shanabrook, and M. E. Twigg, J. Appl. Phys. 85, 2157 (1999).

${ }^{8}$ R. Kaspi and K. R. Evans, J. Cryst. Growth 175/176, 838 (1997).

${ }^{9}$ K. Y. Cheng, K. C. Hsieh, and J. N. Baillargeon, Appl. Phys. Lett. 60, $2892(1992)$

${ }^{10}$ A. G. Norman, S. P. Ahrenkiel, H. Moutinho, M. M. Al-Jassim, A. Mascarenhas, J. Mirecki Millunchick, S. R. Lee, R. D. Twesten, D. M. Follstaedt, J. L Reno, and E. D. Jones, Appl. Phys. Lett. 73, 1844 (1998).

${ }^{11}$ K. C. Hsieh, J. N. Baillargeon, and K. Y. Cheng, Appl. Phys. Lett. 57, 2244 (1990)

${ }^{12}$ R. D. Twesten, D. M. Follstaedt, S. R. Lee, E. D. Jones, J. L. Reno, J. Mirecki Millunchick, A. G. Norman, S. P. Ahrenkiel, and A. Mascarenhas, Phys. Rev. B 60, 13619 (1999).

${ }^{13}$ C. Dorin, J. Mirecki Millunchick, and C. Wauchope, Appl. Phys. Lett. 81, 3368 (2002)

${ }^{14}$ D. W. Stokes, R. L. Forrest, J. H. Li, S. C. Moss, B. Z. Nosho, B. R. Bennett, L. J. Whitman, and M. Goldenberg, J. Appl. Phys. 93, 311 (2003).

${ }^{15}$ H. Bracht, S. P. Nicols, W. Walukiewicz, J. P. Silveira, F. Briones, and E. E. Haller, Nature (London) 408, 69 (2000).

${ }^{16}$ M. J. Cherng, H. R. Jen, C. A. Larsen, G. B. Stringfellow, H. Lundt, and P. C. Taylor, J. Cryst. Growth 77, 408 (1986).

${ }^{17}$ P. F. Fewster, Crit. Rev. Solid State Mater. Sci. 22, 69 (1997).

${ }^{18}$ E. Selvig, B. O. Fimland, T. Skauli, and R. Haakenaasen, J. Cryst. Growth 227-228, 562 (2001).

${ }^{19}$ K. J. Kiely, Philos. Mag. A 60, 321 (1989).

${ }^{20}$ E. S. Tok T. S. Jones, J. H. Neave, J. Zhang, and B. A. Joyce, Appl. Phys. Lett. 71, 3278 (1997).

${ }^{21}$ T. Brown, A. Brown, and G. May, J. Vac. Sci. Technol. B 20, 1771 (2002).

${ }^{22}$ R. Kaspi, J. Cryst. Growth 201/202, 864 (1999).

${ }^{23}$ M. W. Wang, D. A. Collins, T. C. McGill, and R. W. Grant, J. Vac. Sci. Technol. B 11, 1418 (1993).

${ }^{24}$ B. Z. Nosho, B. R. Bennett, L. J. Whitman, and M. Goldenberg, J. Vac. Sci. Technol. B 19, 1626 (2001).

${ }^{25}$ B. Z. Nosho, B. R. Bennett, L. J. Whitman, and M. Goldenberg, Appl. Phys. Lett. 81, 4452 (2002).

${ }^{26}$ C. Dorin and J. Mirecki Millunchick, J. Appl. Phys. 91, 237 (2001).

${ }^{27}$ A. G. Cullis, D. J. Norris, T. Walther, M. A. Migliorato, and M. Hopkinson, Phys. Rev. B 66, 081305 (2002).

${ }^{28}$ O. Dehaese, X. Wallart, and F. Mollot, Appl. Phys. Lett. 66, 52 (1995).

${ }^{29}$ R. Magri and A. Zunger, Phys. Rev. B 65, 165302 (2002).

${ }^{30}$ C. Dorin, J. Mirecki Millunchick, Y. Chen, B. G. Orr, and C. A. Pearson, Appl. Phys. Lett. 79, 4118 (2001).

${ }^{31}$ L. E. Shilkrot, D. J. Srolovitz, and J. Tersoff, Phys. Rev. B 62, 8397 (2000).

${ }^{32}$ H. Asahi, K. Yamamoto, J. Hidaka, J. Satoh, and S. Gonda, J. Cryst. Growth 179, 37 (1997).

${ }^{33}$ J. Steinshnider, J. Harper, M. Weimer, C. H. Lin, S. S. Pei, and D. H. Chow, Phys. Rev. Lett. 85, 4562 (2000). 